\title{
C. VON DER MACHTÜBERNAHME HITLERS BIS ZUR RÖHMI-AFFÄRE
}

(1933/34)

13.

\author{
Hitler - Reichskanzler \\ Gegenbewegung der deutschen Arbeiterklasse \\ Von F. Brand (Berlin)
}

Die neue Regierung Hitler-Papen-Hugenberg-Seldte ist eine offene Kriegserklärung an das arbeitende Volk Deutschlands. Jeder dieser Namen ist ein Programm äußerster Arbeiterfeindlichkeit.

Hitler, der Führer der nationalsozialistischen Terrorformationen, hat die Kanzlerschaft übernommen, um durch rücksichtslose Niederschlagung der Arbeiterklasse und Ausrottung des Kommunismus in Deutschland die Klassendiktatur der Ausbeuterklasse zu sichern. Neben ihm sitzen die Naziführer Frick als Reichsinnenminister, derselbe, der dem Kommunismus eine "Nacht der langen Messer" androhte, und Göring als preußischer Innenminister, der die 100000 Mann preußischer Schupo gegen die Arbeiterklasse einsetzen will.

Papen als Vizekanzler und zugleich Reichskommissar in Preußen, der Herrenklubler, der Führer der "feinen Leute“, ist in den Augen des gesamten werktätigen Volkes der Mann der ungeheuerlichen Notverordnungspolitik, der Hauptverantwortliche für den Lausanner Tributpakt, derselbe, der dem französisch-polnischen Imperialismus eine Verständigung zu Dritt gegen die Sowjetunion anbot.

Hugenberg als Wirtschaftsdiktator, der in seiner Hand die Ministerien der Wirtschaft und Landwirtschaft vereinigt, gilt als der reaktionärste Vertreter der kapitalistischen Scharfmacherinteressen, zugleich als der Führer der monarchistischen Konterrevolution.

Seldte, der Führer des Stahlhelms, als Reichsarbeitsminister ist für die gesamte Arbeiterklasse eine geradezu ungeheuerliche Herausforderung. In seinen Händen schrumpft die gesamte Arbeits- und Sozialpolitik zusammen zu einer Frage des "Arbeitsdienstes", dieser faschistischen Rekrutenschule.

Die Kommunistische Partei Deutschlands brachte zum Ausdruck, was die breitesten Arbeitermassen nicht nur der kommunistischen, sondern auch der sozialdemokratischen und christlichen Arbeiter fühlen und denken, als sie sofort - noch am Nachmittag der Regierungsbildung - die Arbeiterschaft ganz Deutschlands zum stärksten Kampf gegen die faschistische Diktatur aufrief. Unter der Führung der KPD traten am Montagabend die freigewerkschaftlichen und roten Betriebsräte Großberlins über 2000 Mann - zusammen und beschlossen, daß die Belegschaften sofort in den Streik geführt werden sollen. Unter der Führung der Kommunisten bildeten sich am Montag und Dienstag in Berlin, Leipzig, Halle, Kassel und vielen anderen Orten spontane Demonstrationen, welche den Sturz des Hitler-Papen-Kabinetts forderten. Am Dienstagvormittag wurden die ersten Streiks bekannt - die Welle ist ins Rollen gekommen.

Geradezu ungeheuerlich ist der neue Verrat der sozialdemokratischen Partei- und Gewerkschaftsspitzen. Der Reichspräsident, so schreibt der "Vorwärts“, derselbe Hindenburg, welcher dies Kabinett Hitler - Papen - Hugenberg - Seldte eingesetzt hat, 
sei der Garant dafür, daß die Verfassung nicht verletzt werde, und solange die Regierung die Verfassung respektiere, habe die Arbeiterschaft - soll heißen: die SPD- und ADGB-Führung - keinen Anlaß zu irgendwelchen Kampfmaßnahmen! Im Gegenteil, der "Vorwärts" in seiner Montagabendausgabe und die Spitzen der SPD und des $\mathrm{ADGB}$ in der Dienstagmorgenausgabe des "Vorwärts" warnen die Arbeiterschaft vor Aktionen gegen die faschistische Diktatur und verleumden die Kommunistische Partei, daß sie durch „Vorprellen“ nur dem Faschismus helfen könne.

Dieser Verrat der sozialfaschistischen Führer ist noch schlimmer als der Verrat vom 20. Juli, ein geradezu ungeheuerliches Verbrechen an der deutschen Arbeiterklasse. Jetzt, wo es darauf ankommt, der faschistischen Diktatur, noch ehe sie die Machtmittel des Staatsapparats in vollem Unfange einsetzen kann, die Massenaktion der Arbeiterklasse entgegenzustellen, wendet sich die sozialfaschistische Führerclique gegen diesen Kampf. Sie ist dadurch die wertvollste Stütze der Hitler-Papen-Hugenberg-Diktatur geworden! ...

Die Hitler, Frick, Göring sind bereit, alle „sozialistischen“ Verheißungen, die sie ihren Anhängern gemacht haben, zu zerreißen; sie sind bereit, genauso unter das Joch der Tributpolitik zu kriechen, wie die vorhergehenden Regierungen der deutschen Bourgeoisie, und es ist selbstverständlich, daß dabei die Ernüchterung ihrer Anhängerschaft nicht ausbleiben kann. Aber die Arbeiterklasse kann nicht bis dahin warten, sondern muß sofort zu Aktionen übergehen, um ihre Lebensinteressen $z u$ verteidigen und die faschistische Versklavung zu zerbrechen.

Die Regierung Hitler-Hugenberg-Papen ist kein „autoritäres Kabinett" wie die Regierungen Papen und Schleicher; Hindenburg hat ihr diese Vollmachten verweigert und Hitler auf den Weg einer parlamentarischen Mehrheit verwiesen. Diese Mehrheit wird die Regierung - daran ist nach den Erklärungen des Zentrums kein Zweifel mehr möglich - im Reichstag erhalten, und zwar mit Hilfe der Zentrumspartei, die sich bei der Abstimmung über das Mißtrauensvotum der Stimme enthalten will. Es ist möglich, daß bis zu diesem Zeitpunkt, der etwa um die Mitte der nächsten Woche liegen dürfte, die Taktik der Regierung eine möglichst zurückhaltende sein wird wenn nicht die Massenaktionen der Arbeiterklasse unter der Führung der Kommunisten diesen Plan durchkreuzen.

Denn in der Kraft der antifaschistischen Einheitsfront liegt jetzt die nächste Entscheidung. Die Verratspolitik der sozialfaschistischen Führer hat die Lage für die deutsche Arbeiterklasse erschwert. Es kommt alles darauf an, daß in den Betrieben und an den Stempelstellen die Kommunisten diese Sabotage der SPD- und ADGBFührer überwinden und die Massen in den Kampf führen.

[Internationale Pressekorrespondenz Nr. 14 vom 31. Januar 1933, S. 465f.]

14.

Die faschistische Provokation in Deutschland

1. 3.1933

Vernichtungsfeldzug gegen den Kommunismus

..... In Deutschland lieferte die Brandstiftung im Reichstag, diesem „geweihten Hause des Deutschen Volkes", den äußeren Vorwand zur Entfesselung des Vernichtungsfeldzuges gegen den Kommunismus, gegen die ganze revolutionäre Arbeiter- 
bewegung, gegen alles, was nach Freiheit atmet. Auch die deutschen Faschisten wollen den Galgen oder das Henkerbeil zum Symbol ihrer blutigen Herrschaft machen.

Die ganze Gewaltmaschinerie des faschistischen Staates wurde gegen die kommunistische Bewegung in Gang gesetzt. Über das ganze Land ist in der Tat der Belagerungszustand verhängt worden. Die Regierungsgebäude, alle lebenswichtigen $\mathrm{Be}$ triebe, die Gaswerke, Wasserwerke und Elektrizitätswerke sind von Polizeiabteilungen bewacht. Kommunistische Führer und Funktionäre, kommunistische Abgeordnete wurden und werden im ganzen Lande zu Hunderten verhaftet. Verhaftet wurden auch viele linksgerichtete Intellektuelle, Schriftsteller, Wissenschaftler, Rechtsanwälte. Die Gebäude der revolutionären Gewerkschaftsopposition, der Roten Hilfe, der Arbeiterhilfe, aller revolutionären Massenorganisationen wurden polizeilich besetzt. Die kommunistischen Verkehrslokale wurden in Berlin und ganz Preußen überall geschlossen. Die kommunistischen Zeitungen, Zeitschriften, Flugblätter und Plakate wurden auf vier Wochen verboten, die an den Litfaßsäulen angebrachten Wahlplakate der Kommunistischen Partei abgerissen oder überklebt.

Die Terrormaßnahmen richten sich teilweise auch gegen die Sozialdemokratie: Das „Vorwärts"-Gebäude wurde für eine Zeit ebenfalls besetzt, die sozialdemokratische Presse auf zwei Wochen verboten.

Gekrönt wurden diese Terrormaßnahmen, die nur den Auftakt zu einer blutigen Abrechnung mit der revolutionären Bewegung bilden, durch die neue Notverordnung der Regierung „zur Abwehr kommunistischer staatsgefährdender Gewaltakte“ und ,gegen Verrat am deutschen Volk und hochverräterische Umtriebe“. Die Weimarer Verfassung ist damit ein Fetzen Papier geworden. Die letzten kümmerlichen Reste der Freiheit und Unverletzlichkeit der Person und der Wohnung, des Brief-, Post-, Telegraphen- und Fernsprechgeheimnisses, der Freiheit der Meinungsäußerung, einschließlich der Pressefreiheit, der Versammlungs- und Koalitionsfreiheit sind für die Werktätigen vernichtet.

Todesstrafe, Todesstrafe, Todesstrafe: das ist der immer wiederkehrende Refrain dieser ungeheuerlichen Verordnung. Todesstrafen, die bereits für Delikte bei der Zuwiderhandlung gegen behördliche Anordnungen beginnen, und bei Hochverrat, schwerem Aufruhr oder schwerem Landfriedensbruch, bei Giftbeibringung, beim Verrat militärischer Geheimnisse, bei Landesverrat usw. enden. Die Bestimmungen der Notverordnung sind so formuliert, daß sie zu jeder Zeit ohne weiteres gegen alle Kommunisten, gegen jeden revolutionären Arbeiter angewandt werden können. Schildert jemand die Lage in Deutschland, so wie sie ist, - droht ihm die Todesstrafe. Setzt sich ein Arbeiter gegen einen nationalsozialistischen Mordanschlag zur Wehr, die Todesstrafe ist ihm sicher.

Diese Verordnung bedeutet das Verbot der Kommunistischen Partei. Ihre Bestimmungen machen jede legale Tätigkeit der Kommunistischen Partei von vornherein unmöglich. ...

Was veranlaßte den deutschen Faschismus, was veranlaßte die deutschen Nationalsozialisten, diese groß angelegte Provokation in Szene zu setzen? Die Furcht vor der sich anbahnenden Einheitsfront der Arbeiterklasse, die Furcht vor der anhebenden Volksbewegung gegen den Faschismus, die Furcht vor der proletarischen Revolution. Diese Furcht ist der Kitt, der alle Fraktionen der deutschen Bourgeoisie zusammenkittet. Um der nahenden proletarischen Revolution vorzubeugen, sollte die Kommunistische Partei zerschlagen werden, ihre Riesenperipherie unter der Wucht des 
Terrors demoralisiert, die sozialdemokratische Arbeiterschaft eingeschüchtert werden, ja nicht den Durchbruch zur Einheitsfront der Tat zu wagen.

Ein zweiter Grund ist: die wachsende Unzufriedenheit innerhalb des nationalsozialistischen Lagers. Die Massenanhängerschaft der Nationalsozialisten besteht aus ruinierten Kleinbürgern, Kleinbauern, zum Teil aus hungrigen Arbeitslosen. Sie wollen Brot und Arbeit, Hitler und Genossen können ihnen weder Brot noch Arbeit geben. Anstatt dessen bietet man dieser hungrigen Masse das Schauspiel des Vernichtungsfeldzuges gegen die kommunistische Bewegung an. Ein dritter Grund hängt mit den Hegemoniebestrebungen der nationalsozialistischen Bewegung innerhalb der herrschenden Bourgeoisie, innerhalb des faschistischen Systems zusammen. Die nationalsozialistischen Führer sind daran, sich diese Hegemonie zu erkämpfen. Die faschistische Provokation schaffte für sie ein geeignetes Sprungbrett dafür. „Parlamentarisch“: durch gewaltsame Ausschaltung der kommunistischen Fraktion. Außerparlamentarisch: durch Stärkung ihrer Positionen innerhalb des Gewaltapparates des faschistischen Staates.

Die Reichswehr bildet in den Händen Hindenburgs, Papens, Hugenbergs und der Generalität noch immer ein gewisses Gegengewicht gegen die nationalsozialistischen Hegemoniebestrebungen. Die beschleunigte Auffüllung der Polizei durch die nationalsozialistischen Sturmabteilungen, als notwendige Maßnahme gegen die kommunistische Gefahr, die Tatsache, daß die Nationalsozialisten dadurch auch schwere Waffen (Maschinengewehre, Panzerwagen usw.) in die Hände bekommen, bilden in den "Putschplänen" der Nationalsozialisten, für ihren geplanten „Marsch auf Berlin“, einen bedeutenden Aktivposten. ....

Die Ereignisse in Deutschland sind ein Alarmsignal für die Werktätigen aller kapitalistischen Länder. Es geht um ihre eigene Sache! Aktivste Solidarität mit der Kommunistischen Partei Deutschlands, mit der Arbeiterklasse Deutschlands, vor allem durch entschiedene Steigerung des Kampfes gegen die eigene Bourgeoisie, das ist das Gebot der Stunde!

[Rundschau über Politik, Wirtschaft und Arbeiterbewegung Nr. 3 vom 1. März 1933, S. $41 \mathrm{f}$.]

15.

Nach Annahme des „Ermächtigungsgesetzes“

24.3 .1933

Die Annahme des „Ermächtigungsgesetzes“, das der Regierung Hitler-PapenHugenberg auf vier Jahre (bzw. bis zum Auseinanderfallen des gegenwärtigen Kabinetts) völlig unumschränkte Diktaturvollmachten gibt, gewinnt ihre besondere Bedeutung dadurch, daß sowohl das Zentrum wie die Bayrische Volkspartei unter dem starken Druck, nicht zuletzt der Drohungen in der Kanzlerrede, zugestimmt haben. Damit hat sich der Faschismus alle Fraktionen der deutschen Bourgeoisie unterworfen.

.....Von größter Wichtigkeit sind die inneren Gegensätze innerhalb der HitlerPartei selbst, die sich aus dem Klassencharakter der verschiedenen Schichten der nationalsozialistischen Anhängerschaft ergeben. Die ersten Wochen des Jubels über den endlich errungenen Sieg Hitlers sind vorbei; jetzt wachsen die Stimmungen der Kritik, der Unzufriedenheit mit den Ergebnissen. Weder in seiner Potsdamer noch in 
seiner Berliner Regierungserklärung hat Hitler irgendwelche konkreten Andeutungen gemacht, was er den werktätigen Massen seiner Anhänger geben will, die doch jetzt von ihm die Erfüllung all der nationalsozialistischen Verheißungen erwarten. Statt eines Bekenntnisses zu irgendwelchem "deutschen Sozialismus", hat Hitler die „stärkste Förderung der Privatinitiative", d.h. des Kapitalismus, proklamiert; statt irgendwelcher Erleichterungen hat er Maßnahmen in Aussicht gestellt, die „auf keine Popularität rechnen können ". Selbst wenn man die kritische Veranlagung der nationalsozialistischen Anhängerschaft noch so nüchtern einschätzt, angesichts solcher Erklärungen kann eine Enttäuschung kaum ausbleiben. . . .

[Rundschau über Politik, Wirtschaft und Arbeiterbewegung Nr.7 vom 24.März 1933, S.167]

16.

Die Lage in Deutschland
Aus der Resolution des Präsidiums des Exekutiv-Komitees
der Kommunistischen Internationale zum Referat des Genossen Heckert
Angenommen am 1. April 1933

.... Der Sieg Hitlers und die Aufrichtung der Macht der „Nationalsozialisten “ ist durch folgende Umstände ermöglicht worden:

Die deutsche Sozialdemokratie, die die Mehrheit des Proletariats in der NovemberRevolution von 1918 hinter sich hatte, spaltete die Arbeiterklasse und hat, statt die Revolution zur Diktatur des Proletariats und zum Sozialismus vorwärts zu treiben, wie das die Pflicht einer proletarischen Partei gewesen wäre, im Bündnis mit der Bourgeoisie und den wilhelminischen Generalen den Aufstand der revolutionären Massen niedergeschlagen und die tiefe Spaltung der Arbeiterklasse Deutschlands eingeleitet. ...

Das Versailler System plünderte Deutschland aus und beugte die deutschen werktätigen Massen unter das Joch unerträglicher Ausbeutung nicht bloß durch ihr eigenes, sondern auch durch das ausländische Kapital, dem die deutsche Regierung Reparationszahlungen zu leisten hatte. Das Versailler Joch, verstärkt durch das Joch der „eigenen “ deutschen Bourgeoisie, führte zu einer unerhörten Senkung des Lebenshaltungsniveaus des Proletariats und zu einer derartigen Verelendung der Bauernschaft und des städtischen Kleinbürgertums, daß ein Teil der Bauernschaft und des städtischen Kleinbürgertums immer mehr dazu neigte, das Vorkriegsdeutschland, das noch keine allgemeine Krise des Kapitalismus und keine solche Verelendung der Massen kannte wie jetzt, als sein Ideal zu betrachten. Es ist daher begreiflich, daß im Augenblick der stärksten wirtschaftlichen Krise, die die Schwere des durch den Versailler Vertrag auferlegten außenpolitischen Nationaljochs noch steigert, sowie in Verbindung damit, daß das Proletariat aus Verschulden der Sozialdemokratie gespalten und daher nicht stark genug war, um die städtischen kleinbürgerlichen und die bäuerlichen Massen mit sich zu reißen -, daß es in diesem Augenblick zu einem stürmischen Ausbruch des deutschen Nationalismus und Chauvinismus kommen mußte und tatsächlich auch kam, der die politische Stellung der Bourgeoisie bedeutend festigte und die demagogischeste nationalistische Partei - die Partei der "Nationalsozialisten" an die Oberfläche brachte. 
Die kommunistischen Arbeiter organisierten und führten den Kampf gegen die Offensive des Kapitals und des Faschismus. Sie unterstützten jede, auch die geringste Aktion der sozialdem[okratischen] Arbeiter gegen das Kapital, wo immer es zu solchen Aktionen kam. Vom Bestreben geleitet, die revolutionäre Einheit der Arbeiterklasse wieder herzustellen, haben sie bereits lange vor dem Sieg des Faschismus den sozialdemokratischen Arbeitern und den untergeordneten sozialdemokratischen Organisationen wiederholt die Einheitsfront zum Kampf gegen die Bourgeoisie und ihre Lakaien, die Faschisten, vorgeschlagen. Doch die sozialdemokratischen Arbeiter, hinter denen die Mehrheit der Arbeiterklasse Deutschlands steht, haben - gefesselt durch ihre sozialdemokratische Führung, die gegen die revolutionäre Einheitsfront und für die Beibehaltung ihrer reaktionären Einheitsfront mit der Bourgeoisie ist - jedesmal in ihrer großen Masse die Einheitsfront mit den Kommunisten abgelehnt und so den Kampf der Arbeiterklasse gesprengt . . . . .

In Durchführung ihrer Linie des Kampfes um die revolutionäre Einheit der Arbeiterklasse gegen die sozialdemokratische Einheitsfront mit der Bourgeoisie hat die Kommunistische Partei, als einzige revolutionäre Führerin des deutschen Proletariats, ungeachtet des Streikbrechertums der Sozialdemokratie in der Frage der Einheitsfront gegen die Bourgeoisie, die Arbeiterklasse am 20. Juli 1932, als die Faschisten die sozialdemokratische Preußenregierung davonjagten, und am 30. Januar 1933, als Hitler in Deutschland zur Macht kam, zum politischen Generalstreik aufgerufen, und zur Durchführung eines solchen Streiks der Sozialdemokratischen Partei und den reformistischen Gewerkschaften die Einheitsfront vorgeschlagen.

Die Entwicklung des Kampfes gegen Bourgeoisie und Faschismus durch das Proletariat sowie der Generalstreik hätten dazu geführt, daß die schwankenden werktätigen Massen der Bauernschaft und des städtischen Kleinbürgertums zum Proletariat gestoßen wären. Die Sozialdemokratie jedoch unterband, in Fortsetzung ihrer bisherigen Politik sowie in Betreibung der weiteren Arbeitsgemeinschaft mit der Bourgeoisie, die Initiative der Massen durch das Netz der hinter ihr stehenden zentralisierten Organisationen, in erster Linie der reformistischen Gewerkschaften, und verhinderte die Organisierung des Generalstreiks, vereitelte diesen, nicht ohne dadurch der weiteren Offensive der Faschisten gegen das Proletariat unmittelbar Vorschub zu leisten. Als Folge dessen erwies sich die Avantgarde des revolutionären Flügels des deutschen Proletariats - die Kommunistische Partei - der Unterstützung seitens der Mehrheit der Arbeiterklasse beraubt.

Unter solchen Umständen erwies sich das Proletariat in einer Lage, in der es nicht imstande war und tatsächlich auch nicht vermochte, die sofortige und entschlossene Abwehr gegen den Staatsapparat zu organisieren, der in seinen Bestand die Kampforganisationen der faschistischen Bourgeoisie, die Sturmabteilungen, "Stahlhelm“ und Reichswehr zum Kampf gegen das Proletariat mit einbezogen hatte. Die Bourgeoisie vermochte die Staatsgewalt im Lande ohne ernsthaften Widerstand den Händen der Nationalsozialisten zu übergeben, die mit den Mitteln der Provokationen, des blutigen Terrors und politischen Banditentums gegen die Arbeiterklasse vorgehen.

Die Voraussetzungen für den siegreichen Aufstand des Proletariats analysierend, hat Lenin ausgeführt: „Die entscheidende Schlacht kann dann als voll herangereift gelten, wenn sich alle uns feindlichen Klassenkräfte hinreichend verrannt haben, wenn sie sich hinreichend gegenseitig in die Haare geraten sind und sich durch den Kampf, der ihre Kräfte übersteigt, hinreichend geschwächt haben." . . . . 
Die charakteristische Besonderheit der Situation im Moment des Hitler-Umsturzes besteht darin, daß diese Voraussetzungen für den siegreichen Aufstand zu jener Zeit noch nicht auszureifen vermocht hatten - sie waren lediglich im Keimzustand vorhanden.

Was die Avantgarde des Proletariats, die Kommunistische Partei betrifft, so konnte sie, da sie nicht in Abenteurertum verfallen wollte, diesen mangelnden Faktor natürlich nicht durch ihre Handlungen ersetzen.

"Mit der Vorhut allein“, sagt Lenin, „kann man nicht siegen. Die Vorhut allein in den entscheidenden Kampf werfen, solange die ganze Klasse, solange die breiten Massen die Avantgarde nicht direkt unterstützen oder wenigstens eine wohlwollende Neutralität ihr gegenüber üben - wäre nicht nur eine Dummheit, sondern auch ein Verbrechen ..."

Das sind die Umstände, die den Rückzug der Arbeiterklasse und den Sieg der Partei der konterrevolutionären Faschisten in Deutschland bestimmt haben.

Die Errichtung der faschistischen Diktatur in Deutschland ist somit letzten Endes die Folge der sozialdemokratischen Politik der Arbeitsgemeinschaft mit der Bourgeoisie im Verlaufe der ganzen Lebensdauer der Weimarer Republik. . . .

Dadurch aber, daß sich die faschistische Diktatur auf die bewaffneten Banden der Nationalsozialisten und des „Stahlhelm“ stützt, daß sie den Bürgerkrieg gegen die Arbeiterklasse aufnimmt, daß sie alle Rechte des Proletariats aufhebt, zerschlägt sie gleichzeitig auch die sozialdemokratischen Theorien von der Möglichkeit der Eroberung einer parlamentarischen Mehrheit durch Wahlen und von der friedlichen Entwicklung zum Sozialismus, ohne Revolution. Sie zerschlägt die sozialdemokratischen Theorien von der Arbeitsgemeinschaft mit der Bourgeoisie, sowie von der Politik des „kleineren Übels" und macht alle demokratischen Illusionen in den breiten Arbeitermassen zunichte.... .

Gleichzeitig vermag die zügellose faschistische Diktatur Hitlers, die den Bürgerkrieg im Lande entfesselt hat, keine einzige politische und wirtschaftliche Frage des heutigen Deutschlands zu lösen. Not und Elend der Massen nehmen mit jedem Tage mehr zu. Die Lage der Industrie verschlechtert sich, da die abenteuerliche Politik der Regierung lediglich die Schrumpfung sowohl des Binnen- wie auch des Außenmarktes beschleunigt. Es fehlt jegliche Perspektive eines ernsthaften Rückgangs der Erwerbslosigkeit, ja es kann eine solche Perspektive auch gar nicht geben. Es besteht absolut keine Möglichkeit, allen Anhängern der Nationalsozialisten Arbeit und Ämter zu verschaffen. Anstelle der zur Einstellung gelangenden Nationalsozialisten werden andere Arbeiter entlassen werden. Die Verlängerung des Moratoriums bis zum Oktober und die Kontingentierung der landwirtschaftlichen Einfuhrprodukte aus dem Auslande vermag nur auf überaus kurze Zeit eine dünne Schicht der wohlhabendsten Bauern zu befriedigen, vermag aber der Zunahme der Not, des Elends und der Unzufriedenheit der breiten bäuerlichen Massen nicht Einhalt zu gebieten. Durch demagogische Aktionen gegen die Warenhäuser und das jüdische Kapital läßt sich keine Hilfe für das notleidende Kleinbürgertum schaffen, dessen Lage nach Maßgabe des weiteren Rückganges der Kaufkraft des Proletariats sich verschlechtern wird, was eine weitere Schrumpfung des Binnenmarktes nach sich ziehen wird. Die mikroskopische Gabe von Getreide und Speck an die Notleidenden war lediglich ein auf den Stimmenfang berechneter Köder. Die Erhöhung der Erwerbslosenunterstützung um monatlich zwei Mark kann infolge der sich verschlechternden Wirtschaftslage nicht umhin, 
wieder rückgängig gemacht zu werden. Es zeigt sich klar, daß Hitler Deutschland einer Wirtschaftskatastrophe entgegenführt, die sich immer unvermeidlicher gestaltet.....

Die Versuche der Regierung, unter diesen Verhältnissen den Versailler Vertrag zu verletzen und wenigstens durch den Anschluß Österreichs außenpolitische Erfolge zu erzielen, um ihr Ansehen in den Massen zu heben, deren Not und Elend sie nicht $z u$ lindern vermag, wird lediglich zu einer weiteren Verschärfung der gesamten internationalen Situation und zu einem ungeheuren Anwachsen der Kriegsgefahr führen. Jeder neue Tag der Regierung Hitler wird immer klarer den Betrug offenbaren, dem die Massen, die Hitler Gefolgschaft leisteten, zum Opfer gefallen sind. Jeder neue Tag wird immer klarer aufzeigen, daß Hitler-Deutschland in die Katastrophe hineintreibt.

Die augenblickliche Stille nach dem Siege des Faschismus ist nur eine vorübergehende Erscheinung. Der revolutionäre Aufschwung in Deutschland wird trotz des faschistischen Terrors unvermeidlich ansteigen. Die Abwehr der Massen gegen den Faschismus wird zwangsläufig zunehmen. Die Errichtung der offenen faschistischen Diktatur, die alle demokratischen Illusionen in den Massen zunichte macht und die Massen aus dem Einflu $\beta$ der Sozialdemokratie befreit, beschleunigt das Tempo der Entwicklung Deutschlands zur proletarischen Revolution......

[Rundschau über Politik, Wirtschaft und Arbeiterbewegung Nr. 9 rom 2. April 1933, S. 229f.]

17.

Wachsende innere Schwierigkeiten des Faschismus

19. 5.1933

Von E. Varga

Die innerpolitische Lage Deutschlands ist, wenn nicht alle Anzeichen trügen, im Begriff, in ein neues Stadium einzutreten: die Bevölkerung beginnt offen ihre Unzufriedenheit zu zeigen, zu klagen, zu kritisieren, zu nörgeln und zu spotten über das Hitler-Regime! Das ist - nach den breiten Wogen der Hitler-Begeisterung, die in den vergangenen Wochen das ganze Reich überschwemmten und so etwas wie eine Hitler-Psychose erzeugt hatten - eine Tatsache von außerordentlicher Wichtigkeit. Man kann sie überall feststellen, in den Betrieben und auf der Straße, in der Straßenbahn und besonders in den Kaufläden, wo die Preissteigerung wichtigster Lebensmittel sehr erregte Debatten hervorruft. Die Wurzel dieser Mißstimmung ist stets die gleiche: es ist unter Hitler nicht besser, sondern nur noch schlimmer geworden; es ist aber höchste Zeit, daß es endlich besser wird. Diese Gärung ist noch nicht bewußt genug, um in eine klare Ablehnung des faschistischen Regimes zu münden; so weit ist es noch nicht; die meisten, die da kritisieren, haben noch nicht die Illusion aufgegeben, daß es Hitler "vielleicht doch noch schaffen könnte", jedoch meinen sie, es sei jetzt endlich Zeit und wollen nicht mehr lange warten. „Feste haben wir nun genug gefeiert, wir wollen endlich Taten sehen!" das ist die allgemeine Stimmung gerade auch unter den Massen der nationalsozialistischen Anhänger, bis hinein in die Kerntruppen der braunen Armee. . . .

Je größer die inneren Schwierigkeiten des faschistischen Diktaturregimes werden, um so brutaler der Terror gegen die antifaschistische Bewegung. 
$\Lambda$ ber die faschistische Gewaltherrschaft bleibt verstrickt in die inneren Widersprüche zwischen den Interessen und Wünschen der antikapitalistischen Massen und der objektiven Rolle des Faschismus als Schutzgarde eines bankrotten Kapitalismus. Jede seiner Maßnahmen muß diese Widersprüche erneut zurn Ausdruck bringen. Hitlers Appell an die kapitalistische Wirtschaft ist dafür ein klassischer Beweis.

Schon in seiner Tempelhofer Mairede hatte Hitler an die privatkapitalistische Initiative zur Behebung der Arbeitslosigkeit appelliert. Wenige Tage später, am 5. Mai, erneuerte er diese Aufforderung dringender:

"Die Reichsregierung hat alles Interesse daran, daß die Wirtschaft sich jetzt innerlich und ehrlich beruhigt. Alle rigorosen Eingriffe haben zu unterbleiben und werden unterbleiben, so daß die Wirtschaft in der Lage ist, sich auf weite Sicht mit ihren Projekten einzustellen, da die Stabilität ihr die notwendige Gewähr dafür bietet. Die Wirtschaft kann damit beginnen, großzügig zu planen. Der, der schnell und bald damit beginnt, kann der wärmsten moralischen Unterstützung der Reichsregierung versichert sein. Es ist vollkommen fehl am Platze, wenn in der Wirtschaft und in den Wirtschaftskreisen noch irgendeine Nervosität herrscht. Nachdem jetzt auch die Gewerkschaftsaktion durchgeführt ist, sind im Wirtschaftsleben durchaus konsolidierte Verhältnisse eingetreten, so daß sie auf lange Sicht arbeiten kann."

Diese Erklärung, die von größter Bedeutung für die innerpolitische Entwicklung ist, hat eine doppelte Wirkung: eine auf die kapitalistische Wirtschaft und eine andere auf die antikapitalistische Massenstimmung. Die Kapitalisten fragen zurück: Wie sollen wir auf weite Sicht planen, wenn doch noch alles im Dunkeln liegt? Was wird aus der Mark? Wird die Mark dem Dollar und dem Pfund folgen? Wie sollen die riesig angeschwollenen öffentlichen Ausgaben gedeckt werden? Wie soll die "Arbeitsbeschaffung " finanziert werden? Muß nicht die neue Zinskonversion den Kreditmarkt aufs neue schwer stören? Was wird aus den exportierenden Industrien? Wie soll der Binnenmarkt „belebt“ werden, wenn die Konsumkraft der Massen weiter sinkt? Wie kann man uns zumuten, neue Investitionen zu machen, wenn der Absatz ununterbrochen weiter zurückgeht und die bisherigen Werksanlagen zu drei Vierteln ungenutzt liegen? Und vor allem: Wird die Hitlerpartei der erneut wachsenden antikapitalistischen Kräfte Herr werden, die sich vor allem in den Betrieben geltend machen und sogar die nationalsozialistischen Betriebszellen zu Lohnforderungen vorwärtstreiben? Das sind die Gründe der vorhandenen „Nervosität“, gegen die sich Hitler wendet. Kann sich die Regierung einbilden, alle diese Momente mit einer solchen Erklärung totschlagen zu können? Es ist anzunehmen, daß dieser neue Appell Hitlers an seine kapitalistischen Auftraggeber keinen größeren Erfolg haben wird als alle seine vorangegangenen Ermahnungen.

Auf die antikapitalistisch eingestellten Massen der nationalsozialistischen Anhängerschaft aber muß diese Erklärung Hitlers umgekehrt im höchsten Masse ernüchternd und aufreizend wirken. Keinerlei Eingriffe mehr? Also soll alles so bleiben wie es bisher war? Ist das die Beseitigung des kapitalistischen Schmarotzertums, die Hitler versprochen?

Hitler garantiert den Kapitalisten die „Stabilität“ ihrer $\Lambda$ usbeutungswirtschaft. Wie verträgt sich das mit Hitlers Verheißung auf den „deutschen Sozialismus"? Wo bleibt die „Brechung der Zinsknechtschaft“? Wo die Enteignung der Trusts, der Bank- und Börsenfürsten, der Warenhäuser? Wo bleibt die Beseitigung der erdrückenden Steuerlast? Will Hitler sein ganzes Programm zerreißen? 
Für die Arbeiterschaft ist insbesondere der Satz über den Sinn der Gewerkschaftsaktion von größter Bedeutung. Dadurch seien jetzt die Wirtschaftsverhältnisse konsolidiert! Haben nicht die Naziführer den Arbeitern erklärt, die "Gleichschaltung“ der Gewerkschaften sei notwendig, um die Mißwirtschaft der sozialdemokratischen Bonzen zu beseitigen? Jetzt aber hat Hitler selbst den wahren Zweck enthüllt: Konsolidierung des kapitalistischen Systems! Jetzt droht die Zerschlagung des Tariflohnsystems und seine Ersetzung durch den „elastischen Leistungslohn“, jetzt droht das Verbot des Streiks! Das also ist Hitlers "deutscher Sozialismus"?

Eine neue Erklärung des preußischen Ministerpräsidenten Göring, die am 9. Mai veröffentlicht wurde, richtete sich vor allem gegen die Aktionen des gewerblichen Mittelstandes. Sie stellt fest, daß sich der nationalsozialistische „Kampfbund für den gewerblichen Mittelstand " wiederholt Eingriffe in Betriebe erlaubt habe, d. h. gegen die Warenhäuser und Filialgeschäfte. Solche Eingriffe seien künftig strengstens verboten und würden bestraft werden. Ein ähnliches Verbot richtet sich gegen den „Kampfbund für deutsche Kultur" und seine „Reichszentrale für das Theaterwesen“, d.h. gegen die nationalsozialistischen Schauspieler, Musiker usw. Derartige Kampfbünde, erklärt Göring, dürften nicht in Interessenvertretungen ausarten, sondern müßten im Gegenteil die Interessenvertretungen zurückdrängen und durch eine allgemeine Staatsauffassung ersetzen. Ja, wozu haben denn dann die Handwerker und Krämer, die Leute vom Theater und Film Hitler zugejubelt, wenn nicht, weil sie eine bessere Wahrnehmung ihrer Interessen [er]hofften? Das soll nun alles nicht wahr sein? Wo bleibt denn da die Erfüllung der nationalsozialistischen Versprechungen?

Dazu kommt endlich noch die Erklärung der Reichsregierung, daß alle Steuern und Steuerrückstände rücksichtslos eingetrieben werden sollen! Eine kalte Dusche für die Bauern und die Mittelständler, denen die Nazis die Streichung der Steuerrückstände versprochen und eine Senkung der Steuerlasten in Aussicht gestellt hatten. Wozu haben sie denn dann die "nationale Revolution" gemacht?

Das sind die großen inneren Schwierigkeiten des faschistischen Regimes, die sich jetzt in zunehmendem $\mathrm{Maße}$ geltend machen und aus denen der Faschismus keinen Ausweg findet. Mit verschärftem Terror versucht er davon abzulenken, indem er alle unzufriedenen Elemente als „kommunistisch“ verketzert. Aber damit wird er wenig Erfolg haben. Was heute erst in den Anfängen sich zeigt, wird wachsen und in zunehmendem Maße den Boden günstiger machen für die Arbeit der Kommunisten. Das ist der Hintergrund für die hysterische Tobsucht der Kieler Hitlerrede.

[Rundschau über Politik, Wirtschaft und Arbeiterbewegung Nr. 14 vom 19. Mai 1933, S. $425 f$.

\section{8.}

....W Während die internationale bürgerliche Presse noch mit der Ausdeutung der Erfurter Rede Hitlers beschäftigt war, worin er die Aufhebung der Klassengegensätze in Deutschland feierlich verkündete, da kamen schon einzelne Nachrichten darüber, wie die neue Volksgemeinschaft geschaffen wird: die Verhaftung von katholischen Geistlichen in der Pfalz, die Auflösung einzelner Stahlhelmortsgruppen. Aber das 
waren nur kleine Vorspiele, denen allzubald viel wichtigere Ereignisse folgen sollten. Am 21. Juni erfuhr die staunende Welt, daß die deutschnationalen Kampforganisationen und Jugendverbände gewaltsam, mit einem plötzlich durchgeführten Überfall, aufgelöst wurden. Gleichzeitig wurde bekanntgegeben, daß sich der Stahlhelm einer neuen Amputation unterziehen und hinnehmen mußte, daß er in einen nationalsozialistischen Veteranenverein umgewandelt werde. Damit wurden dem Partner der "nationalen Revolution“ tödliche Wunden beigebracht. Der Nationalsozialismus gab sich jedoch nicht zufrieden. Am 22. Juni wurde der längst erwartete Schlag gegen die Sozialdemokratie geführt, die treu nach der Pfeife Hitlers tanzte und seinerzeit kein Wort des Protestes fand, als die kommunistischen Mandate kassiert wurden: es wurden ihre Landtags- und Reichstagsmandate annulliert, - freilich nicht aus Angst vor Löbe, Ebert jun. und Scillat, sondern aus Angst, daß sich um sie Kristallisationspunkte einer wirklichen Opposition bilden könnten. Und schließlich, um das Werk zu krönen, wurde zu Wochenend der Kommissar für sämtliche evangelischen Landeskirchen Preußens ernannt, um die „vorhandene Verwirrung" zu beseitigen, d.h. den Widerstand gegen den Nazipfarrer Müller durch diktatorische Maßnahmen zu brechen. Der Rücktritt Hugenbergs und die „Selbstauflösung“ der Deutschnationalen Partei setzen gewissermaßen nur den Schlußpunkt hinter diese Ereignisse.

Die Nazipresse jubiliert. Sie bezeichnet diese Vorgänge als den Übergang zu der „zweiten Stufe“ der nationalsozialistischen Revolution, als eine Etappe zur „Totalität" - ein neues Wort im Wörterbuch der Nazis, das bedeuten soll, daß der ganze Staat ein einheitliches nationalsozialistisches Gepräge erhält, keine Machtpositionen sich in anderen als in Nazihänden befinden dürfen. Sie weisen auf Italien hin, wo der Staat gleichbedeutend mit dem Faschismus ist und das nun auch in Deutschland eintreten soll, da doch der deutsche Nationalsozialismus eigentlich nach den Prinzipien des italienischen Faschismus aufgebaut sei. Dieses Vorgehen wäre also erst die richtige Entfaltung des Nationalsozialismus. Und so behauptet denn auch die Nazipresse, daß die Verkrüppelung des Stahlhelms, die Zerstörung der deutschnationalen Kampforganisationen, die Liquidierung der SPD-Parlamentsfraktion, schließlich die wirkliche „Gleichschaltung " der evangelischen Kirche - daß all das für einen neuen unerhörten Aufstieg der nationalsozialistischen Bewegung zeugen würde.

In Wirklichkeit verhält sich die Sache ganz umgekehrt. Was beweist hier das Beispiel Italiens? Die Entwicklung des italienischen Faschismus zeigt, daß in dem Maße, wie seine Massenbasis schwand, sein Drang nach Alleinherrschaft stärker wurde. Mussolini hatte kein Programm der "Totalität“, er kümmerte sich den Teufel um jedes Programm. Jahre hindurch arbeitete er, ja kämpfte er sogar mit dem Parlament - in einer Sache war er unerbittlich: in der Niederhaltung der Arbeiterklasse. Der Not gehorchend, nicht dem eigenen Triebe, ging der italienische Faschismus gegen das Parlament vor und entschied sich für die "Totalität“. Es ist möglich, daß als Hitler, Göring und Frick in den historischen Januartagen sich Hindenburg, Papen, Seldte und Hugenberg gegenüber ehrenwörtlich verpflichteten, keine Herrschaft nach italienischem Muster zu errichten, sie in Wirklichkeit schon damals an die sogenannte Totalität dachten. Es ist das möglich. Aber das beweist nur, daß sich die nationalsozialistischen Führer schon damals bewußt waren, daß die von ihnen entfachte und noch mehr zu entfachende Begeisterung sehr bald abflauen wird, und dann gibt es nur eine Rettung für sie: die nackte Gewalt, der rücksichtsloseste Terror. Der Hinweis auf Italien zeugt im besten Falle dafür, daß sich in Deutschland die Dinge viel rascher 
entwickeln; eine Entwicklung, die dort Jahre brauchte, wird hier in Monaten zurückgelegt. .... .

[Rundschau über Politik, Wirtschaft und Arbeiterbewegung Nr. 22 vom 30. Juni 193j, S. 717]

19.

Die gegenwärtige Lage in Deutschland und die Aufgaben der KPD

Resolution, beschlossen vom Politbüro des $\mathrm{ZK}$ der KPD

Diese Resolution vutrde vom Politbüro des $Z K$ der KPD in seiner Sitzung vom 10. Oktober 1933, also einige Tage vor dem Austritt Hitler-Deutschlands aus dem $V$ ölkerbund, beschlossen. Die neuesten Ereignisse unterstreichen die Richtigkeit der in der Resolution enthaltenen Analyse und Perspektive.

Vor acht Monaten, unter den Verhältnissen der außerordentlichen Verschärfung der politischen und wirtschaftlichen Lage in Deutschland, übergab die deutsche Bourgeoisie die Verwirklichung der offenen faschistischen Diktatur an Hitler und seine „nationalsozialistische“ Partei. Die acht Monate des Bestehens der Blut- und Hungerregierung Hitler-Göring-Goebbels haben im vollen Umfange bestätigt, daß die brutale hemmungslose faschistische Diktatur keine einzige politische und wirtschaftliche Frage des heutigen Deutschlands zu lösen vermag. . .

Durch ihre Abenteurerpolitik treibt sie alle inneren und äußeren Widersprüche des deutschen Kapitalismus auf die Spitze und führt Deutschland der Katastrophe entgegen. In der vom Faschismus vollkommen entrechteten Arbeiterklasse wächst, ungeachtet des schlimmsten, blutigsten Terrors, ein ungeheuerer revolutionärer Aufschwung heran. Die Massen des städtischen Kleinbürgertums und der Bauernschaft beginnen bereits zu durchschauen, daß sie von den Nationalsozialisten betrogen wurden.....

[Rundschau über Politik, Wirtschaft und Arbeiterbewegung Nr. 40 rom 27. Oktober 1933, S. 1541$]$

20.

Der deutsche Faschismus in der Sackgasse

a) Der Austritt Deutschlands aus dem Völkerbund Von E. Varga

Die deutsche faschistische Presse berichtet uns Tag für Tag über die Festlichkeiten, die in Deutschland stattfinden. Jeden Tag werden auf irgendeinem Platze in Deutschland Zehntausende, Hunderttausende zu einer Feier zusammengetrieben. Einmal ist es der Parteitag der Nationalsozialisten, ein anderes Mal der Bauerntag, Feste der SA, militärische Paraden, Enthüllungen von Denkmälern usw. Fast jeden Tag spricht Hitler zu den Massen, suggeriert ihnen, daß nunmehr alles gut gehen wird. Diese krampfhaften Bemühungen, eine Feststimmung zu erzeugen, immer wieder durch neue Injektionen das rasch schwindende Vertrauen der kleinbürgerlichen Massen in den Faschismus wieder aufleben zu machen, sind charakteristisch für das gegenwärtige Stadium des deutschen Faschismus. 
Denn daß der deutsche Faschismus in rapidem Tempo seine Anziehungskraft auf die breiten Massen verliert, darüber kann kein Zweifel bestehen. Uns war es von vorneherein klar - wir haben dies bereits vor einem halben Jahre an dieser Stelle ausgeführt -, daß der Faschismus unfähig sei, seiner kleinbürgerlichen Gefolgschaft in Stadt und Land eine reale Besserung ihrer Lage zu bringen. Dies folgt zwangsläufig aus dem Klassenwesen des faschistischen Regimes als Werkzeug des Monopolkapitals zur Sicherung und Wiederherstellung ihres durch die Krise geschmälerten Profits, als Werkzeug, um einen kapitalistischen Ausweg aus der Krise auf Kosten aller Werktätigen zu finden. Das faschistische Regime braucht Festlichkeiten, braucht immer neue, die Volksmassen aufpeitschende ideologische Mittel, um ihre ausschließlich den Interessen der herrschenden Klasse dienende Wirtschaftspolitik zu verhüllen, den Prozeß der Enttäuschung und Abwendung der Massen vom Faschismus zu verlangsamen.

Dies ist der allgemeine Hintergrund zum Verständnis des theatralischen Effekts, mit dem der Austritt Deutschlands aus dem Völkerbund und das Einstellen seiner Teilnahme an der Abrüstungskonferenz erfolgte.... .

Die rasch wachsende Unzufriedenheit der Massen mit der Wirtschaftspolitik Hitlers z.u dämpfen, ist zweifellos einer der Hauptbeweggründe für den Austritt Deutschlands aus dem Völkerbund und das Verlassen der Abrüstungskonferenz.

Da die Lage der werktätigen Massen: Arbeiter, armen und kleinen Bauern auf dem Lande, Angestellten, Beamten, Handwerker in der Stadt, mit Ausnahme der Prätorianergarde Hitlers, sich durchgehends verschlechtert hat, mußte eine große nationale Geste gemacht werden, um das schwindende Vertrauen der Massen zeitweilig auf chauvinistischer Basis wieder [zu] festigen.

[Rundschau über Politik, Wirtschaft und Arbeiterbewegung Nr. 44 vom 18. November 1933]

21.

Das XIII. Plenum des Exekutivkomitees der Kommunistischen Internationale Referat des Genossen Pieck über die KPD

\section{....III. Politik und Perspektive der faschistischen Diktatur}

... Die Bemühungen der faschistischen Diktatur, ihren Klassencharakter durch die Ideologie der „Volksgemeinschaft der Nation“, der Ersetzung der Klassen durch die "gleichberechtigte Vertretung von Berufsständen" $\mathrm{zu}$ verstecken, werden von allen Parteien der II. Internationale unterstützt. Die österreichische „linke“ Sozialdemokratie, mit Otto Bauer an der Spitze, schuf die Theorie von der faschistischen Diktatur als der „Diktatur des Kleinbürgertums". Trotzki bezeichnete die faschistische Diktatur als die "kleinbürgerliche Konterrevolution".

Diese Theorie ist für die Arbeiterklasse sehr gefährlich. Sie bedeutet:

1. eine Unterstützung der faschistischen Demagogie, die die Diktatur der Thyssen und Krupp als „Herrschaft des gesamten Volkes mit dem von der Vorsehung berufenen Führer" zu verschleiern sucht;

2. eine nachträgliche Rechtfertigung der Arbeitsgemeinschaftspolitik der Sozialdemokratie mit der Bourgeoisie, denn wenn die faschistische Diktatur als selbständige Kraft der Bourgeoisie gegenübersteht, dann - so sagen die Wels und Konsorten - war 
es prinzipiell richtig, mit Brüning und Teilen der Bourgeoisie eine Koalition gegen den Faschismus einzugehen;

3. bedeutet diese Theorie den Versuch, die sozialdemokratischen Arbeiter von jeglichem Kampf gegen die gesamte Bourgeoisie und gegen jede bürgerliche Herrschaftsform abzuhalten.

Zu einem alten Artikel im „Manchester Guardian“, der kürzlich in Berlin in einer Neuauflage verbreitet wurde und in dem die Ablösung der Hitler-Diktatur durch eine Militärdiktatur als wahrscheinlich bezeichnet wird, schrieb die Sozialdemokratie, daß eine solche Militärdiktatur, eventuell unter Schleicher, der Arbeiterschaft eine größere Handlungsfreiheit geben würde und eine Stufe sei, dem Sozialismus näher zu kommen. Die Neumann-Remmele-Gruppe hat die sozialdemokratische Theorie von der "Diktatur des Kleinbürgertums" gewandelt in eine „Diktatur des Lumpenproletariats“ und sie ergänzt durch die Theorie von einem "Systemwechsel“, von der Ablösung der Herrschaft der Bourgeoisie durch die Herrschaft der zum Lumpenproletariat herabsinkenden bürgerlichen Gesellschaft. Diese Theorie leugnet die Tatsache, dal3 in der faschistischen Diktatur der Inhalt der Klassenherrschaft der Bourgeoisie derselbe geblieben ist wie in der „Weimarer Demokratie“ und führt in der Konsequenz zı einer Gegenüberstellung der Herrschaftsform zwischen bürgerlicher Demokratie und faschistischer Diktatur. Sie bedeutet die Rechtfertigung der Arbeitsgemeinschaftspolitik der Sozialdemokratie und leugnet das Herauswachsen der faschistischen Diktatur aus der bürgerlichen Demokratie.

Die Entwicklung in Dentschland bestätigt die Feststellung des XII. EKKI-Plenums, daß man von einer klassischen Form der faschistischen Diktatur nicht sprechen kann und sich gegen jede mechanische Gleichsetzung und oberflächlichen Vergleiche der faschistischen Diktatur in Deutschland und Italien wenden muß. Die Form und die Dauer der faschistischen Diktatur sind abhängig von den objektiven und subjektiven Faktoren der gesamten Entwicklung. Gerade für die Einschätzung der Perspelativen der faschistischen Diktatur in Deutschland ist die Kenntnis ihrer entscheidenden Besonderheiten notwendig. - Diese sind:

1. Die faschistische Diktatur in Deutschland wurde aufgerichtet gegen eine unbesiegte Arbeiterklasse, die zwar vorübergehend zurückwich, aber sich wieder zum Angriff sammelt; sie wurde aufgerichtet in einem im Weltkriege besiegten Lande, dessen Bourgeoisie alle Opfer des Krieges und der Reparationen auf die Werktätigen abwälzt. Der Versuch der Rückeroberung der alten Machtstellung innerhalb der imperialistischen Welt, der einer der Faktoren ist, die die Bourgeoisie zur Machtübergabe an Hitler veranlaßte, zwingt die deutsche Bourgeoisie, ihre Vorbereitungen zum Kriege in beschleunigtem 'Tempo durchzuführen.

2. Die faschistische Diktatur in Deutschland wurde aufgerichtet in einem Lande weitgehender Erschütterungen des Kapitalismus, in dem industriellsten Lande Europas.

3. Die Diktatur wurde aufgerichtet in einem Lande, dessen Arbeiterschaft über die stärkste Klassenorganisation in den kapitalistischen Ländern mit einer jahrzehntelangen Tradition und einem marxistisch geschulten Kader von Funktionären der KPD und RGO verfügte, in einem Lande, in dem die Gefahr einer raschen Abwendung des Kleinbürgertums und der kleinen und mittleren Bauern von der faschistischen Diktatur, besonders unter den Auswirkungen eines revolutionären Aufschwungs, für die Bourgeoisie besonders stark ist.....

[Rundschau über Politik, Wirtschaft und Arbeiterbewegung Nr. 4 vom 13.Januar 1934, S.121] 
.. Der Faschismus ist keine unvermeidliche faschistische Periode, denn zum wissenschaftlichen Begriff einer geschichtlichen Periode gehört die Entfaltung neuer Produktionsverhältnisse. Die Periode des Industriekapitalismus bedeutete die Befreiung der Entwicklung der Wirtschaft von den Schranken des Feudalismus, die Periode des Monopolkapitalismus bedeutete die monopolistische Organisierung der Kräfte, die vom Industriekapitalismus geschaffen worden waren. Diese Aufgabe hatte ohne den Faschismus derart ungeheure Ausmaße erreicht, daß der Monopolkapitalismus selbst aufhörte, notwendig und unvermeidlich zu sein, sondern zu einem Hindernis der geschichtlichen Entwicklung wurde. Da nun der Monopolkapitalismus aufhörte, notwendig zu sein, der Sozialismus aber notwendig und möglich wurde, wofür der Monopolkapitalismus alle Voraussetzungen schuf, hat der Faschismus keinerlei geschichtliche unvermeidliche Funktionen vor sich, ohne deren Erfüllung sich die Geschichte nicht weiterbewegen könnte.

Dutzende Wirtschafter haben sich bemüht, das wirtschaftliche Geheimnis des italienischen l'aschismus zu erraten, herauszufinden, worin denn der Korporativstaat besteht, jene wirtschaftliche Neuordnung, von der die Faschisten schwatzen. Wenn sich die deutschen Faschisten ausreden können, indem sie darauf hinweisen, daß sie erst ein Jahr alt seien, daß man deshalb keine Abrechnung machen könne, so können die italienischen Faschisten diese Antwort nicht geben. Sie sind zehn Jahre an der Macht, und man kann ihnen bereits eine Rechnung vorlegen. Selbst wenn es unmöglich sein sollte, eine endgültige Durchführung ihres Programms zu fordern, so sind sie verpflichtet zu zeigen, wohin sie gehen. Ihr Schrank springt aber ganz einfach auf. Sie gehen wirtschaftlich zur gleichen Verstärkung der Herrschaft des kapitalistischen Monopols und zum gleichen Verfall wie der Kapitalismus in der ganzen Welt. Die Krise hat in Italien zu den gleichen Folgen geführt, wie in allen anderen kapitalistischen Ländern, mit dem Unterschied, daß die faschistische Diktatur es noch mehr gestattete, die Volksmassen ohne Ümschweife auszubeuten.

Der Faschismus stellt nicht eine geschichtlich unvermeidliche Etappe dar.

Wo das Proletariat eine kräftige revolutionäre Vorhut herauskristallisiert hat, wo es dieser Vorhut gelingen wird, um sich die Mehrheit der Arbeiterklasse zu scharen, sich an die Spitze der Armen in Stadt und Land zu stellen, das Kleinbürgertum zu spalten, dort wird das Proletariat siegen, wie es in Rußland gesiegt hat, ohne durch clas Fegefeuer des Faschismus hindurchzugehen. Die faschistische Diktatur ist ein letzter Versuch, diesen unvermeidlichen Sieg des Proletariats zu verschieben, ihn weiter hinauszuziehen, und wo der Faschismus dank besonderer politischer Verhältnissen siegt, dort besteht seine einzige geschichtliche Funktion darin, in den Arbeitermassen die reformistisch-demokratischen Illusionen zu töten, in der Arbeiterklasse die Weichherzigkeit zu töten, die Bedingungen zu schaffen, unter denen jene ehernen Scharen herausgeschmiedet werden, die imstande sind, die Macht zu ergreifen und sie mit ehernen Armen festzuhalten, indern sie den verzweifelten Widerstand der Bourgeoisie erdrücken. Der Platz des Faschismus in der Geschichte ist der letzte verzweifelte Kampf, der sich der Weltrevolution nähert, der Kampf, in dem er untergehen wird, und dies nicht „irgendwann“..... Die Worte des Genossen Stalin, 
„daß der Gedanke des Sturmes im Bewußtsein der Massen heranreift", das ist die Perspektive, von der ausgehend die Zukunft des Faschismus beurteilt werden muß.

Unfähig, auch nur einen einzigen Widerspruch aufzulösen, die Menschheit auch nur einen einzigen Schritt vorwärtszubringen, ist er nur zu einem fähig, den Untergang der Bourgeoisie katastrophaler, blutiger zu gestalten. Es geht nicht um die Bestimmung der kalendermäßigen Fristen dieses Untergangs. Es geht um das Begreifen dessen, daß diese Todeskrämpfe des Weltkapitals, die den Volksmassen schwere Nöte bereiten können, in der nächsten Geschichtsperiode mit der Zertrümmerung der faschistischen Bourgeoisie enden werden, um das Begreifen dessen, da $\beta$ es in der Geschichte keinen Platz für eine ganze Periode des Faschismus gibt.

[Rundschau über Politik, Wirtschaft und Arbeiterbewegung Nr. 29 vom 9.Mai 1934, S.1105]

23.

Der blutige 30. Juni in Deutschland

Am 30. Juni hat Hitler die „Volksgemeinschaft" verwirklicht. Er ließ seine nächsten Parteigenossen und Kampfgefährten füsilieren. Am 30. Juni hat Hitler den Klassenkampf liquidiert. Er hat den Bürgerkrieg gegen seine eigenen Massenorganisationen brutal, bestialisch, niederträchtig durchgeführt. Er hat auch die nationalsozialistische „Kameradschaft" verwirklicht, indem er mit einem Teil seiner Partei den anderen Teil hinrichten und niedertrampeln ließ. Er hat das nationalsozialistische Prinzip "Treue um Treue" im Geiste des echten Frontsoldatentums zu seinem logischen Ende geführt, indem er Röhm, Heines, Ernst und die übrigen Führer seiner SA durch seine SS-Banditen niedermetzeln ließ. Das Führerprinzip wurde ebenfalls verwirklicht. Die Unterführer wollten ihren „Führer" stürzen, und der Führer bewarf sogar die Leichen seiner Partei- und Kampfgenossen mit Dreck und Schmutz, jene, die er der deutschen Jugend als alte Kämpfer, als wirkliche „Herrenmenschen“ hingestellt hat.....

Nur eine zum Tode verurteilte Klasse, nur eine zum T'ode verurteilte Gesellschaftsordnung, kann solche Vertreter, solche „Helden“, solche Führer haben. Verlumpt, verfault, wie der deutsche Monopolkapitalismus ist, verlumpt, zersetzt, verfault, wie die faschistische Diktatur ist, im Schmutz, in Dreck, im Blute watend, wie die herrschenden Klassen Deutschlands, so sehen die Repräsentanten dieses „Systems“ aus! Einen Zuhälter, Horst Wessel, macht man zum Nationalhelden, sie alle sind dieses ihres Nationalhelden würdig.

Der 30. Juni hat die ganze tiefe Krise der faschistischen Diktatur offenbart, und der 30. Juni ist der Anfang vom Ende der faschistischen Diktatur in ihrer nationalsozialistischen Prägung. Die wirtschaftlichen, sozialen, innen- und außenpolitischen Krisenschläge kamen am 30. Juni zum Ausbruch, aber dieser 30. Juni bedeutet gleichzeitig den krampfhaft verzweifelten Versuch des deutschen Monopolkapitals, seine faschistische Diktatur zu retten und seine offen terroristische Diktatur auf neuer Grundlage umzubilden.

Der Staat besteht aus Abteilungen von bewaffneten Menschen mit solchen Attributen wie Gefängnissen - hat uns Engels gelehrt. Am 30. Juni trat der Staat des Dritten Reiches nackt, ohne phrasenhafte Verschleierung in Erscheinung. Belagerungszu- 
stand, d. h. die Reichswehr, die Polizei und ausgesuchte Abteilungen der SS wurden gegen die SA eingesetzt, SA-Führer erschossen, ins Gefängnis geworfen, SA-Stabsquartiere besetzt, die SA auf Urlaub geschickt, ihr das Uniformtragen verboten und - wie blutig komisch das klingen mag - ihr das Tragen des „Ehrendolches" bis auf weiters untersagt. Die SA wird gesäubert, "gereinigt", Zehntausende und Hunderttausende "alter und neuer Kämpfer des Nationalsozialismus“ werden verjagt, die Kerntruppe des Dritten Reiches wird mit Schmutz und Dreck beworfen.

So wurde ein Widerspruch des Dritten Reiches, der faschistischen Diktatur, nicht gelöst, sondern auf einer höheren Stufe reproduziert. Dieser Widerspruch bestand darin, daß sich das deutsche Monopolkapital in der NSDAP eine Waffe zur Durchführung der faschistischen Diktatur schuf, daß das deutsche Monopolkapital durch die NSDAP an die breiten kleinbürgerlichen, bäuerlichen, plebejischen und auch lumpenproletarischen Massen appellierte, um seine offen-terroristische Diktatur gegen das Proletariat aufrichten zu können. Dieser Widerspruch bestand darin, daß es dem Faschismus in Deutschland wie in keinem anderen Lande gelang, sich eine breite kleinbürgerliche, bäuerliche, plebejische, lumpenproletarische Massenbasis zu schaffen, um - an die Macht gelangt - die offen-terroristische Diktatur der reaktionärsten, am meisten chauvinistischen, am meisten imperialistischen Elemente des Finanzkapitals durchzuführen. Nunmehr werden die kleinbürgerlichen Schichten auf dem Lande und in der Stadt - insbesondere die aktivistischen Elemente der SA und NSBO - mit ihrer Unzufriedenheit und Empörung zu einer antifaschistischen Kraft werden, die wir als Verbündete gewinnen können ...

Die SA war eine Prätorianergarde, eine Parteiarmee im Dienste des Finanzkapitals. Nach derMachtergreifung durch Hitler strömten Hundert- und aber Hunderttausende in die Reihen der SA, die bald 2,5 bis 3 Millionen umfaßte. Die monopolistische Großbourgeoisie war gezwungen, in ihrem Bürgerkrieg gegen das Proletariat und um den imperialistischen Krieg vorzubereiten, an die breiten Massen zu appellieren. Mit den Millionenmassen strömte aber auch die Enttäuschung, die Unzufriedenheit, die Empörung, die Rebellion der kleinbürgerlichen Massen, der plebejischen Schichten und sogar gewisser rückständiger Schichten des Proletariats in die Reihen der SA. Die Röhm, Ernst, Heines und die übrigen Condottieri des Faschismus träumten vom Sturze Hitlers, von der Errichtung eines SA-Staates. Sie wollen sich diese unzufriedenen Stimmungen der empörten Massen - wenn auch in verzerrter Form zunutze machen. Sie konnten selbstverständlich nicht verstehen, daß in der Periode des Monopolkapitalismus die kleinbürgerlichen, plebejischen, lumpenproletarischen Schichten, unter deren sozialem Druck sie trotz ihres Condottieriwesens standen, zwischen den zwei Mühlsteinen der modernen Gesellschaft, zwischen Bourgeoisie und Proletariat, keine selbständige Rolle spielen konnten.

Die monopolistische Großbourgeoisie hat die Rebellion der kleinbürgerlichen Schichten, insoweit diese in der Spitze der SA zum Ausdruck kam, mit Hilfe der Reichswehr, Polizei und SS blutig niedergeschlagen, und sie konnte sie niederschlagen, weil das Proletariat durch seine Massenaktionen die Führung über diese Schichten noch nicht erringen konnte. Die Hitler, Göring, Goebbels waren bei lieser Operation nur die Lakaien des reaktionärsten Finanzkapitals, sie waren das Aushängeschild, um nicht sofort mit voller Klarheit die blutige, eklige Fratze des Großkapitals und Junkertums sehen zu lassen. 
Hitler war in Neudeck und hat durch Hindenburg die Befehle von OldenburgJanuschau und der ostelbischen Junker demütig entgegengenommen. Von Neudeck fuhr er zu Krupp und hat von diesem die Befehle der Schwerindustrie und des Reichsstandes der deutschen Industrie unterwürfig in Empfang genommen. Auf Neudeck und Essen folgte dann München. In München und Berlin wurden die in Neudeck und Essen erhaltenen Befehle prompt durchgeführt.

[Rundschau über Politik, Wirtschaft und Arbeiterbewegung Nr. 39 vom 5. Juli 1934, S. $1541 \mathrm{f}$. 